\title{
UCRL-JRNL-204975
}

LA WRENCE LIWEAMCAE NATIONAL LABOAATOAY

\section{Experimental Studies of Electrons in a Heavy-Ion Beam}

A. W. Molvik, P. A. Seidl, F. M. Bieniosek, R. H. Cohen, A. Faltens, A. Friedman, M. Kireeff Covo, S. M. Lund

June 23, 2004

Nuclear Instruments and Methods A 
This document was prepared as an account of work sponsored by an agency of the United States Government. Neither the United States Government nor the University of California nor any of their employees, makes any warranty, express or implied, or assumes any legal liability or responsibility for the accuracy, completeness, or usefulness of any information, apparatus, product, or process disclosed, or represents that its use would not infringe privately owned rights. Reference herein to any specific commercial product, process, or service by trade name, trademark, manufacturer, or otherwise, does not necessarily constitute or imply its endorsement, recommendation, or favoring by the United States Government or the University of California. The views and opinions of authors expressed herein do not necessarily state or reflect those of the United States Government or the University of California, and shall not be used for advertising or product endorsement purposes. 


\title{
Experimental Studies of Electrons in a Heavy-Ion Beam
}

\author{
A. W. Molvik ${ }^{\mathrm{a}}$, P. A. Seidl ${ }^{\mathrm{b}}$, F. M. Bieniosek ${ }^{\mathrm{b}}$, R. H. Cohen ${ }^{\mathrm{a}}$, A. Faltens ${ }^{\mathrm{b}}$, \\ A. Friedman ${ }^{\mathrm{a}}$, M. Kireeff Covo ${ }^{\mathrm{a}}$, S. M. Lund ${ }^{\mathrm{a}}$, L. Prost ${ }^{\mathrm{b}}$ \\ Heavy Ion Fusion Virtual National Laboratory, \\ ${ }^{a}$ Lawrence Livermore National Laboratory, Livermore, CA 94550, USA. \\ ${ }^{b}$ Lawrence Berkeley National Laboratory, Berkeley CA 9472-82010, USA
}

\begin{abstract}
Electron cloud effects, ECEs, are normally a problem only in ring accelerators. However, heavy-ion induction linacs for inertial fusion energy have an economic incentive to fit beam tubes tightly to intense beams. This places them at risk from electron clouds produced by emission of electrons and gas from walls. We have measured electron and gas emission from $1 \mathrm{MeV} \mathrm{K}{ }^{+}$impact on surfaces near grazing incidence on the High-Current Experiment (HCX) at LBNL. Electron emission coefficients reach *values of 130, whereas gas desorption coefficients are near $10^{4}$. Mitigation techniques are being studied: A bead-blasted rough surface reduces electron emission by a factor of 10 and gas desorption by a factor of 2. Diagnostics are installed on HCX, between and within quadrupole magnets, to measure the beam halo loss, net charge and expelled ions, from which we infer gas density, electron trapping, and the effects of mitigation techniques. Here we discuss a new diagnostic technique that measures gas pressure and electron ionization rates within quadrupole magnets during the beam transit.
\end{abstract}

PACS: 29.27.Bd, 34.50.Dy, 52.70.NC, 52.58.Hm

Keywords: Electron cloud, pressure rise, pressure measurement

\section{Introduction}

Electron cloud effects (ECEs) [1] and beam-induced pressure rises [2], that are frequently observed to limit the performance of colliders and high-intensity rings, are a concern for future high-intensity heavy ion accelerators such as envisioned in Heavy Ion Inertial Fusion (HIF) [3].

The cost of accelerators for HIF can be reduced by fitting beam tubes more tightly to beams. This places them at risk from gas desorption runaway, and from electron clouds produced by secondary electrons and ionization of gas. We are engaged in an experimental and theoretical program to measure, understand, and model these effects in heavy-ion accelerators [4,5]. In this paper, we review measurements of ion induced electron emission and gas desorption for ions near grazing incidence, discuss a mitigation technique [6], discuss measurements using diagnostics inside quadrupole magnets to measure local densities of gas, and the rate of electron generation from ionization of gas.

On HCX we are using a $1 \mathrm{MeV}, 180 \mathrm{~mA}, \mathrm{~K}^{+}$ion beam to study transport [7], beam induced electron emission and gas desorption [6], and electron cloud and gas effects in magnetic quadrupoles. The beam has a space-charge potential of $\sim 2 \mathrm{kV}$, rise and fall times of $1 \mu \mathrm{s}$, and a flattop duration of $4 \mu \mathrm{s}$, repeated at $10 \mathrm{~s}$ intervals. An aperture can be inserted at the D2 diagnostic region, immediately preceding the magnetic quadrupoles, to reduce the beam current to $25 \mathrm{~mA}$ and $\sim 300 \mathrm{~V}$ beam potential. Electron transit times

${ }^{*}$ Corresponding author e-mail address: molvik1@1lnl.gov (A. W. Molvik) 
between walls are in the range of $7 \mathrm{~ns}$ ( $20 \mathrm{~ns}$ if apertured) for an unneutralized beam, almost 3 orders of magnitude shorter than the flattop duration. This enables exploration of unique electron trapping regimes: multipactor trapping will not occur during the flattop, and trailing edge multipactor is not an issue because any electrons generated will be lost before the next pulse in $\sim 10 \mathrm{~s}$. However electrons, emitted from the wall under beam bombardment, will be trapped during the current rise at the beam head. Ionization of gas by the beam also generates electrons that are deeply trapped; the associated ions from gas are expelled by the positive beam potential in $\leq 1 \mu \mathrm{s}$.

\section{Mitigation of electron and gas emission}

Electron emission and gas desorption coefficients $\eta_{\mathrm{e}}$ and $\eta_{0}$ respectively, due to ion bombardment of metal surfaces near grazing incidence, have been measured with the Gas-Electron Source Diagnostic (GESD). This information enables us to interpret electron emission currents from electrodes in beam tubes in terms of the beam-halo loss that caused the emission, and to infer the resulting gas desorption. The GESD is also useful for studying mitigation techniques. We find that $\eta_{\mathrm{e}} \sim 10^{2}$ and $\eta_{0} \sim 10^{4}$ for $1 \mathrm{MeV} \mathrm{K}$ ions incident on stainless steel [6]. The electron emission coefficient is shown in Fig. 1(a). The angular dependence of the electron emission coefficient $\eta_{\mathrm{e}}$ is observed to scale as $\eta_{\mathrm{e}} \propto \mathrm{d} / \cos (\theta)$, where $\mathrm{d} / \cos (\theta)$ is the ion path length through a thin $\mathrm{d} \approx 2 \mathrm{~nm}$ thick surface layer (where the beam-induced electrons originate). Similar scaling is observed at higher ion energies by Thieberger [8].

The gas desorption coefficient $\eta_{0}$, is measured from the pressure rise after a pulse. The GESD pumps out through the 0.3 by $2.5 \mathrm{~cm}$ entrance aperture, plus a $1 \mathrm{~cm}$ diameter hole, giving a pump-out time constant of $0.3 \mathrm{sec}$, long enough for an ion gauge to determine the peak pressure, but short compared with the $10 \mathrm{~s}$ before the next pulse. The less than $1 / \cos (\theta)$ dependence of gas desorption indicates that it is not only from layers of gas adsorbed on the surface (Fig. 1(b)). Similar scaling is observed at higher ion energies by Mahner [9].

Halo loss in magnetic quadrupoles occurs at the maximum excursion of the beam envelope, near the major diameter; without ion scattering any electrons emitted from the wall are confined close to the wall (every $90 \mathrm{deg}$ ) by the quadrupole magnetic field. Ion scattering from the surface exacerbates the emission and desorption, not only allowing ions to impact a surface multiple times, but allowing them to impact the wall in regions from which the quadrupole magnetic field passes through the beam. We have evaluated ion scattering using the SRIM (TRIM) Monte Carlo code [10], which predicts that 60$70 \%$ of ions reflect (called backscatter in the code) when incident at 88-89 deg. from normal [6]. The scattered ions are mostly within $\sim 10 \mathrm{deg}$ of the initial direction but a few scatter by up to $90 \mathrm{deg}$. The scattered ions enable electrons from the wall to fill the entire quadrupole magnet in the simulation of Cohen [11]. This broad angular distribution of scattered ions also complicates suppression of electron emission from grids and electrodes of diagnostics. These could be shielded from near-grazing halo ions by low beam scrapers, but scrapers high enough to remove the scattered ions reduce the beam aperture significantly and are still only partially effective.

Ion scattering decreases rapidly away from grazing incidence, by an e-fold every $8.5^{\circ}$ [6]. Based on this observation, we tried roughening a surface using glass beam blasting. 
This was intended to greatly reduce the fraction of the area on which an ion can impinge at grazing incidence, resulting in most ions striking the surface at angles significantly away from grazing.

The surface looks like it has undergone fine-grained sandblasting. The approximate scale length of the roughness of the surface is $100 \mu \mathrm{m}$. We characterized the target roughness, before installing it in the Gas-Electron Source Diagnostic (GESD) apparatus, by measuring the spot size of reflected light from a laser at near normal incidence onto the target. The bright spot indicated half angles of $17 \mathrm{deg}$, out to about $23 \mathrm{deg}$ for fainter light. However, this technique over emphasizes the bottoms of craters, which may be relatively flat, whereas ions near grazing incidence will preferentially strike the rim of the craters, which are likely to be steeper. Since electron emission from a smooth surface was well fit by 6.06/cos(theta), an emission coefficient of 12.8 implies at angle of $62 \mathrm{deg}$ from normal. The change in gas desorption is consistent with such an angle, from extrapolating the measured dependence on angle back to about $60 \mathrm{deg}$, although we don't yet have a model for how this should vary. The effect on ion scattering is based entirely on the SRIM code at present. At $60 \mathrm{deg}$. the predicted ion backscattering is 3\%, down a factor of 20 from the $60-70 \%$ near grazing incidence.

\section{Gas and electrons in magnetic quadrupoles}

Fig. 2 shows the HCX in the region of four magnetic quadrupoles. To the left is the D2 diagnostic region between 10 electrostatic quadrupoles and the 4 magnetic quadrupoles. Each magnetic quadrupole has $30 \mathrm{~cm}$ long magnetic field coils in a $47 \mathrm{~cm}$ length elliptical tube that has minor and major inner radii of $3 \mathrm{~cm}$ and $5 \mathrm{~cm}$ respectively. Between each pair of magnets, and after the last one, diagnostic access is provided in a 5 $\mathrm{cm}$ gap, each with 7 ports. Arrays of diagnostics are mounted, on octagonal tubes that fit the elliptical beam tube bore, in the third and fourth quadrupoles. A gap of about $0.75 \mathrm{~cm}$ annular space is provided between the octagonal diagnostics mounting tube and the elliptical magnet bore for the recessed diagnostics and cables, Fig. 3. These diagnostics include capacitive electrodes to measure the net beam charge, electrodes shielded from the beam electric field by grids to measure the current of expelled ions from gas ionization in the fourth quadrupole, and flush electrodes running the length of the octagonal tube in the third quadrupole divided azimuthally into two per quadrant. Electron-clearing ring electrodes are inserted in the drift regions between quadrupole magnets, and can be biased to $+10 \mathrm{kV}$ to remove electrons from the drift regions between magnets. Another ring electrode surrounding the beam between the exit of the last quadrupole magnet and the downstream diagnostics can be biased to $-10 \mathrm{kV}$ to suppress beam-induced electrons generated on the end structures from reaching the magnets.

For initial tests of the diagnostics in magnetic quadrupoles, we aperture the beam at D2 to reduce the beam halo near the walls. This reduces the full beam current of $180 \mathrm{~mA}$ to about $25 \mathrm{~mA}$.

One pair of gridded electrodes is located on the minor axis of the octagonal tube in the fourth magnet where the quadrupole magnetic field is tangent to the electrode surface, such that it can suppress electron emission or collection by the electrode. The grids are electroplated mesh from Buckbee Mears, 35 mesh $/ \mathrm{cm}, 88 \%$ transmission, 5 $\mu \mathrm{m}$ thick nickel, that are spot welded around the periphery of the aperture $(0.7 \times 10 \mathrm{~cm})$. Two 
grids, separated by $0.25 \mathrm{~mm}$ are used to improve the shielding of the electric field of the beam. Bench tests performed by pulsing a square wave onto a $2.5 \mathrm{~cm}$ diameter metal rod through the center of the diagnostics tube demonstrated that the shielding reduced the pickup by the shielded electrodes by a factor of $490 \pm 60$ times compared to an unshielded capacitive electrode scaled to the same aperture area.

The gridded electrode is biased at $-50 \mathrm{~V}$. The current varies by only $12 \%$ as the bias is varied from -30 to $-80 \mathrm{~V}$; this saturation of the current indicates that we have a reliable measurement. The quadrupole magnetic field provides magnetic insulation to prevent electrons from flowing between the grids and the electrode. Ions, however, are driven across the magnetic field (B' $\sim 8 \mathrm{~T} / \mathrm{m}$ ) by the beam potential. Singly ionized argon can be expelled across the field with a partially-neutralized beam potential as low as $35 \mathrm{~V}$, or with an unneutralized, apertured beam potential of $300 \mathrm{~V}$, ions as low in mass as $5 \mathrm{amu}$ can reach the electrode.

With the apertured beam, we observe currents that ramp from 0.6 to $\sim 2 \mu \mathrm{A}$ to each gridded electrode, Fig. 3(a). This shape is reasonable: the initial value appears to represent the base pressure, and the ramping could be due to desorbed gas reaching the beam within the fourth magnet. We test this hypothesis by injecting argon gas into the gap between magnets 3 and 4, thereby varying the base pressure with a known gas. We find that the initial current, at the beginning of the beam pulse, increases with the gas pressure; and that the ramping of current, after the initial fast rise, continues to rise by $\sim 1.5 \mu \mathrm{A}$ at the end of each pulse. This is consistent with the beam loss to the wall (and the resulting gas desorption) not varying with gas pressure, Fig. 3. We calculate the gas pressure at the center of the gridded electrodes within magnet 4 , from measurements made with a cold cathode ionization gauge between magnets 3 and 4 and a Bayard-Alpert ionization gauge in D-End. We assume that the pressure decreases linearly through the fourth magnet, and determine that the gridded electrode is $37 \%$ of the way upstream towards drift region (c). We correct for argon gas with the manufacturer's correction factor of 0.8 relative to air. Then

$\mathrm{P}($ effective $)=0.37\left[\mathrm{P}_{\text {base }}+0.8\left(\mathrm{P}_{34}-\mathrm{P}_{\text {base }}\right)-\mathrm{P}_{\text {end }}\right]+\mathrm{P}_{\text {end }}$

For Fig. 3(b), we convert pressure to the density of molecules $\left(3.3 \times 10^{16}\right.$ molecules $/\left(\mathrm{cm}^{3}\right.$ torr).

We estimate the fractional volume of the beam that expels ions into a gridded electrode: the electrode aperture is $0.7 \mathrm{~cm} \times 9.8 \mathrm{~cm}$ long at a minor radius of $2.4 \mathrm{~cm}$. From an envelope code, we determine that the major radius is approximately twice the minor radius; we arbitrarily choose $4 \mathrm{~cm}$ and $2 \mathrm{~cm}$. Then the pie-slice of beam viewed is 0.023 of the total beam volume in that $9.8 \mathrm{~cm}$ length. From a particle balance $\mathrm{dn}_{\mathrm{i}} / \mathrm{dt}=\mathrm{n}_{\mathrm{b}} \mathrm{n}_{0} \mathrm{~V}_{\mathrm{b}} \sigma_{\mathrm{I}}-\mathrm{n}_{\mathrm{i}} / \tau_{\mathrm{d}}$ where $\tau_{\mathrm{d}}$ is the time for an electron to drift the length of a magnet. We multiply $\mathrm{dn}_{\mathrm{i}} / \mathrm{dt}$ by an electron charge and by the volume of beam viewed $\left(5.7 \mathrm{~cm}^{3}\right.$ for beam dimensions corresponding to a beam density of $1.55 \times 10^{8} \mathrm{~cm}^{-3}$ ), and divide by the square of the beam transmission ( $\mathrm{T}=0.88$ through each of two grids) to calculate the measured expelled ion current. Solving for the ionization plus charge exchange cross sections, we obtain $\sigma_{\text {I }+\mathrm{cx}}=\mathrm{I}_{\mathrm{GIC}} /\left(\mathrm{q} \mathrm{n}_{\mathrm{b}} \mathrm{V}_{\text {viewed }} \mathrm{n}_{0} \mathrm{v}_{\mathrm{b}} \mathrm{T}^{2}\right)=1.4 \times 10^{-14} \mathrm{~cm}^{2}$.

This is near, or exceeds, the upper limit of plausible values for the cross section (sum of ionization of gas by beam impact and charge exchange cross sections). One uncertainty is that the ionization gauges are not calibrated. More importantly, we need to evaluate the 
possibility of other mechanisms contributing to the measured current, thereby increasing the inferred cross section; for example, beam-gas photon emission could generate photoelectrons at electrodes and scattered beam ions could cause more electron emission at the electrodes; if electron emission is not completely suppressed by the quadrupole magnetic field, this would increase the current and the inferred ionization cross section. In future work, we will be addressing these issues.

Despite possible large uncertainties in measuring the cross-section, we have demonstrated a valid pressure measurement that we have approximately calibrated. A more accurate calibration can be achieved in the future by calibrating the ion gauges and by using a static gas fill to eliminate the pressure drop across the fourth magnet. We have developed an in-situ fast ionization gauge that measures gas density within an ion beam. It is applicable to space-charge dominated beams with relatively high fill factor, so that cold ions can be expelled across the quadrupole magnetic field by the beam potential, and a beam duration that is longer than the cold ion expulsion time.

This diagnostic also provides a nearly direct measurement of the generation rate of electrons by beam impact ionization of gas. The measured ion current differs from the electron ionization rate because charge exchange produces a cold ion, but no electron. In general, charge exchange cross sections are smaller than ionization cross section, particularly for non-resonant interactions (i.e., the beam ion is not the same element as the gas atom or molecule). Therefore, we expect the electron ionization rate to be within a factor of 2 of the ion expulsion rate: we will ignore this correction, as well as the other possible contributors to the measured current, in addition to expelled ion.

To analyze the particle balance of electrons, we evaluate the electron lifetime $\tau_{\mathrm{d}}=(\mathrm{L}) /\left(\mathrm{v}_{\mathrm{ExB}}+\mathrm{V}_{\nabla \mathrm{B}}\right)$.

where $\mathrm{L}=$ Length of quad. We recognize that the ExB drift velocity is proportional to the beam potential, which will decrease if electrons partially neutralize the beam. Similarly, the $\nabla \mathrm{B}$ drift (including curvature drift) depends on the energy of the electrons perpendicular and parallel to the magnetic field. We therefore multiply $\mathrm{v}_{\mathrm{d}}=\mathrm{L} / \tau_{\mathrm{d}}$ by the factor $\left(n_{b}-n_{e}\right) / n_{b}$. Eq. 2 then becomes

$\mathrm{dn}_{\mathrm{e}} / \mathrm{dt}=\mathrm{n}_{\mathrm{b}} \mathrm{n}_{0} \mathrm{v}_{\mathrm{b}} \sigma_{\mathrm{I}}-\mathrm{n}_{\mathrm{e}}\left[\left(\mathrm{n}_{\mathrm{b}}-\mathrm{n}_{\mathrm{e}}\right) / \mathrm{n}_{\mathrm{b}} \tau_{\mathrm{d}}\right]$

Solving this in equilibrium $\left(\mathrm{dn}_{\mathrm{e}} / \mathrm{dt}=0\right)$, we obtain a quadratic equation for $\mathrm{n}_{\mathrm{e}}$ which has two roots:

$\mathrm{n}_{\mathrm{e}} / \mathrm{n}_{\mathrm{b}}=\mathrm{n}_{0} \mathrm{v}_{\mathrm{b}} \sigma_{\mathrm{I}} \tau_{\mathrm{d}} \leq$ few $\%$,

and

$\mathrm{n}_{\mathrm{e}} / \mathrm{n}_{\mathrm{b}}=1$.

The implication of the first root is that the electron drift velocity rapidly transports them out of a quadrupole magnet into a drift region where either a clearing electrode [12] or an acceleration gap can remove the electrons fast enough that they do not build up. The second root has $n_{e} / n_{b}=1$ so the beam potential is zero and the drift velocities are therefore zero.

This final discussion was meant to be a first attempt to use direct measurements of gas density and the electron ionization rate within a quadrupole magnet. While the initial conclusions are uncertain, we now have a clearer view of the paths forward in electron and gas studies for HIF. 


\section{Acknowledgements}

We are grateful to Ralph Hipple, William Strelo, Ron Beggs, Tak Katayanagi, Gary Ritchie, Craig Rogers, and Ed Romero for excellent technical support. This work was performed under the auspices of the U.S. Department of Energy by University of California, Lawrence Livermore National Laboratory under contract No. W-7405-Eng48, and by University of California, Lawrence Berkeley National Laboratory under Contract DE-AC03-76F00098.

\section{References}

[1] http://wwwslap.cern.ch/collective/ecloud02/ proceedings/index.html

[2] W. Y. Zhang, "ICFA Workshop on Beam Induced Pressure Rise in Rings," http://www.c-ad.bnl.gov/icfa (2003).

[3] R. O. Bangerter, Phil. Trans. R. Soc. Lond. A 357, 575 (1999).

[4] A. W. Molvik, R. Cohen, A. Friedman, S. Lund, et al., "Initial Experimental Studies of Electron Accumulation in a Heavy-Ion Beam," Proc. Of the 2003 Particle Accelerator Conference, p. 312 (IEEE, 2003).

[5] R. H. Cohen, A. Friedman, S. M. Lund, A. W. Molvik, M. Furman, J.-L. Vay, and P. Stoltz, Proc. Of the 2003 Particle Accelerator Conference, p. 132 (IEEE, 2003).

[6] A. W. Molvik, M. Kireeff Covo, F. M. Bieniosek, L. Prost, P. A. Seidl, D. Baca, A. Coorey, and A. Sakumi, "Gas desorption and electron emission for $1 \mathrm{MeV}$ potassium ion bombardment of stainless steel," Submitted to Phys. Rev. Special Topics Accelerators and Beams, (2004).

[7] L. Prost, HIF-04.

[8] P. Thieberger, A. L. Hanson, D. B. Steski, S. Y.Z. V. Zajic, and H.Ludewig, Phys. Rev. A. 61, 042901 (2000).

[9] E. Mahner, J. Hansen, J.-M. Laurent, and N. Madsen, Rhys. Rev. Special Topics Accelerators and Beams 6, 013201 (2003).

[10] J. F. Ziegler, http://www.srim.org/.

[11] R. H. Cohen, et al., HIF-04.

[12] A. W. Molvik, et al., Ecloud04. 

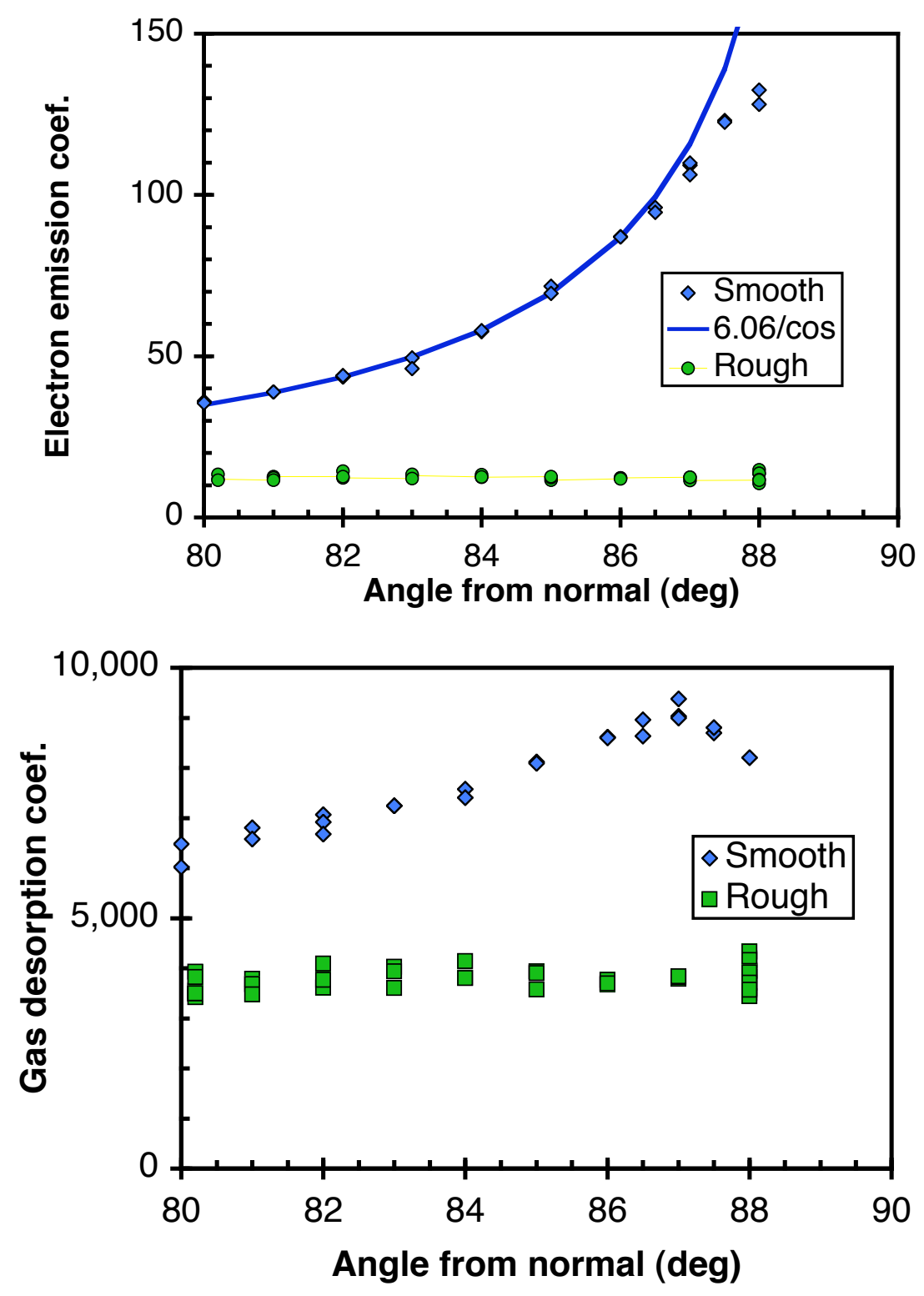

Fig. 1. (a) Electron emission coefficient as a function of angle of incidence, measured from normal to the stainless steel target. [blue-diamonds] Data from a smooth target. The blue line, given by $6.06 / \cos (\theta)$, is a fit to the data between $80^{\circ}$ and $86^{\circ}$. [Green circles] Data from a surface roughened by bead blasting. (b) [blue diamonds] Gas desorption coefficient data from a smooth surface; [green squares] similar data from a bead-blasted surface. 


\section{Suppressor}

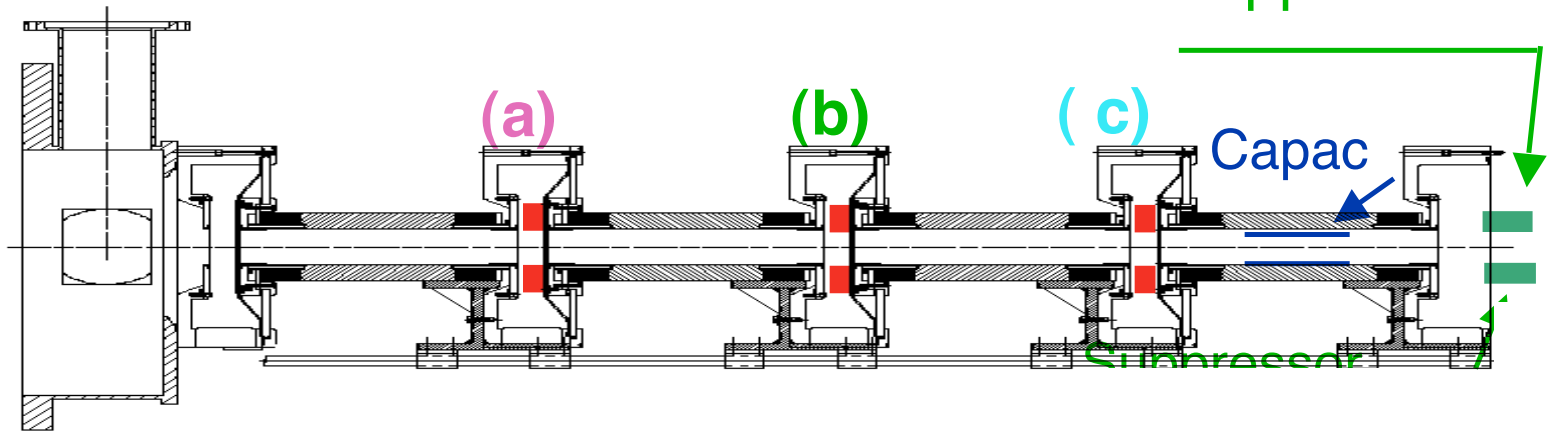

Fig. 2. Magnetic quadrupole region of HCX, from D2 diagnostic region on the left to the D-End diagnostic region beginning on the right. The half lattice length is $0.52 \mathrm{~m}$. Clearing electrodes $\mathrm{a}, \mathrm{b}$, and $\mathrm{c}$ are shown in the drift regions between each pair of quadrupoles. A suppressor electrode prevents beam induced electron emission, from structures hit by beam in D-End, from reaching the quadrupole magnets. Diagnostics are mounted on the outside of octagonal tubes, in the bores of the third and fourth magnets, and are either flush with, or recessed behind, the inner wall of the octagonal tubes. 

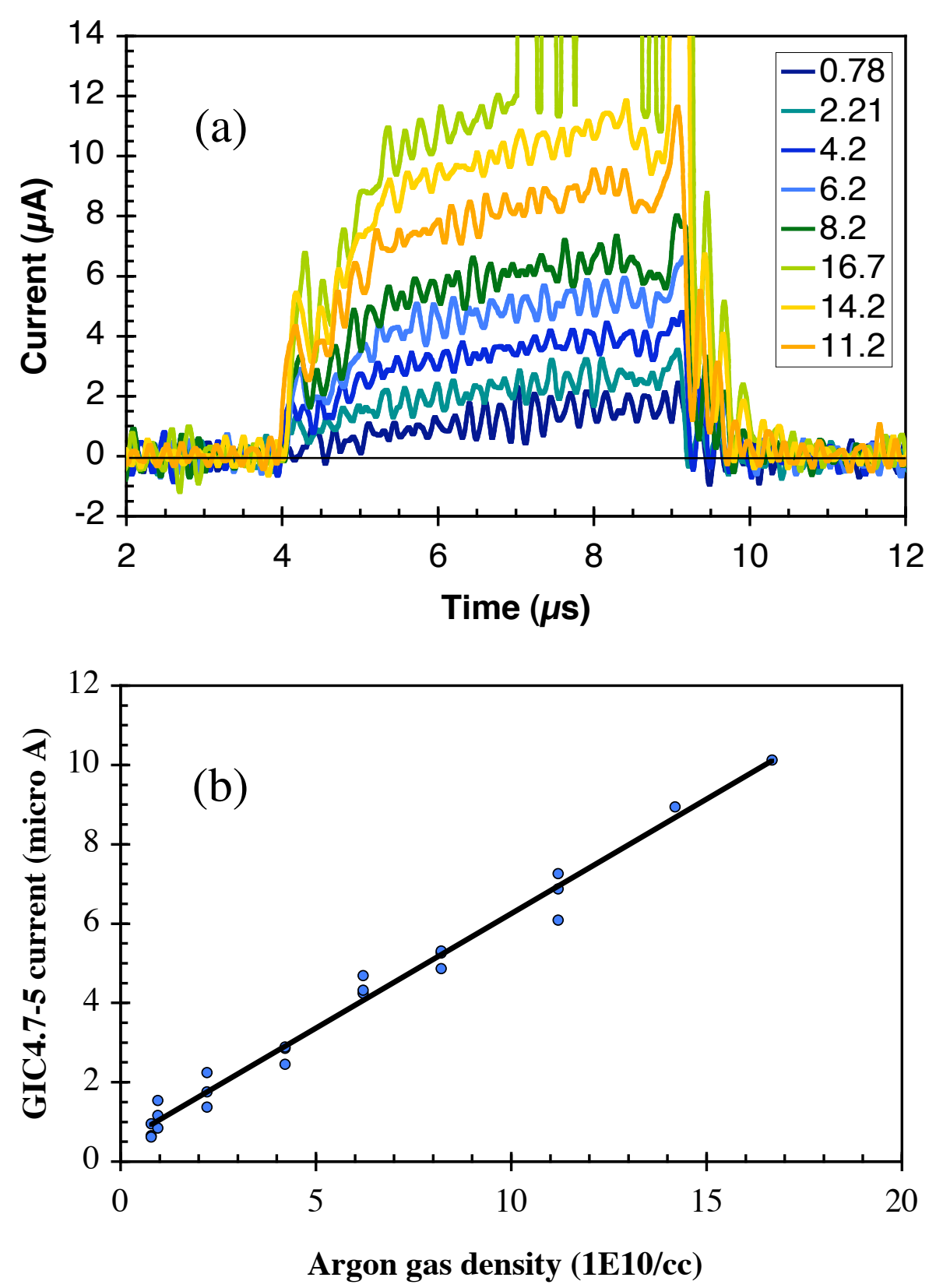

Fig. 3. (a) Current to a grid-shielded electrode versus time. The legend gives the gas density, calculated from ion gauge measurements as described in text. (b) The initial current increases linearly with the background pressure, indicating that it provides a measurement of gas density within a magnetic quadrupole. 